\title{
Incipience and development of spall fracture in 30KHGSA steel, $\alpha \leftrightarrow \varepsilon$ transformation, spall fracture and damage recovery
}

\author{
Svetlana Malyugina*, Sergey Mokrushin, Anna Mayorova, Dmitry Kazakov, Oleg Kozelkov, Alexander Pavlenko, \\ Dmitry Belyaev \\ RFNC-FNIITF, 456770. Snezhinsk, Russia
}

\begin{abstract}
Results of investigation into strength and elasto-plastic properties of 30KhGSA steel are presented. The investigation was performed with planar samples using submicrosecond shockwave length technique. Shockwave amplitude varied in the range from 4 to $26 \mathrm{GPa}$ and controlled with $0.2 \%$ accuracy. Steel sample temperature varied in the range from $-90{ }^{\circ} \mathrm{C}$ to $670{ }^{\circ} \mathrm{C}$. Stress wave profiles in the samples were registered with VISAR and PDV interferometers having nanosecond time resolution and measurement error better than $0.8 \%$. Special attention was payed to the study of spall fracture incipience and development processes as well as damage recovery (healing), and shockwave passing through the damaged region in steel samples. Relaxation characteristics of dynamic elastic limit were determined. Spall strength magnitude versus strain rate and temperature characteristics was determined. The impact of polymorphic $\alpha \leftrightarrow \varepsilon$ transition in 30KhGSA steel on spall strength was studied.
\end{abstract}

\section{Introduction}

Chromium-silicon-manganese steel 30KhGSA (chromansil), owing to its high strength and good weldability, is widely used in different industries, especially in aircraft industry. Besides, this steel, like iron, exhibits wide spectrum of behavioral features and properties in dynamic processes. This allows it to be considered as an important model material when studying shock wave impacts on solids. The shock wave structure in solids is defined by processes of their viscoelastoplastic deformation, phase transitions, and fracture incipience and development kinetics [1-3].

This paper presents the results of investigation into strength and elasto-plastic properties of 30KhGSA alloyed steel in submicrosecond shockwave length range. During the measurements shock waves impacted the samples under controlled loading conditions in the pressure range from 4 to $26 \mathrm{GPa}$. The impact pulse length varied from 0.1 to $1.2 \mu \mathrm{sec}$. Relaxation properties of the dynamic elastic limit were determined. Spall strength magnitude versus deformation velocity was determined, the temperature effect and polymorphic $\alpha \leftrightarrow \varepsilon$ transition impact on spall strength at deformation velocities of $10^{4}-10^{6} \mathrm{~s}^{-1}$ were studied.

Particular attention was given to studying spall fracture incipience and development processes, as highvelocity deformation of materials is a multi-stage process [4-5] accompanied by defect incipience, formation of material microdamages (pores or cracks), and merging of damages in a single main crack. The backward process, referred to as damaged medium compacting (healing) often occurs with compression wave closing cracks and pores up to continuous medium formation. Then, the paper presents the results of investigations into damage recovery (healing), and shock wave passing through the damage area in the steel samples.

\section{The material and experimental set up}

The steel 30KhGSA refers to the class of alloyed structural steel. Table 1 gives steel chemical composition (in $\%$ by mass).

Table 1. Chemical composition of 30KhGSA steel.

\begin{tabular}{|c|c|c|c|c|c|c|c|}
\hline $\boldsymbol{F e}$ & $\boldsymbol{C}$ & $\boldsymbol{C r}$ & $\boldsymbol{M n}$ & $\boldsymbol{S i}$ & $\boldsymbol{N i}$ & $\boldsymbol{P}$ & $\boldsymbol{S}$ \\
\hline basis & 0.32 & 0.94 & 1.0 & 1.05 & 0.15 & 0.014 & 0.007 \\
\hline $\boldsymbol{C} \boldsymbol{u}$ & $\boldsymbol{M o}$ & $\boldsymbol{V}$ & $\boldsymbol{T i}$ & $\boldsymbol{W}$ & \\
\cline { 1 - 5 } 0.1 & 0.02 & 0.005 & 0.025 & 0.011 &
\end{tabular}

The basic dopants for 30KhGSA steel are chrome, manganese, and silicon.

The samples of nominal thickness $0.5,1,2,4,8$, $10 \mathrm{~mm}$ were cut from a cylindrical rod $45 \mathrm{~mm}$ in diameter. The rod was heat treated in order to refine grains and to obtain a martensite structure. The grain size was $(24 \pm 7) \mu \mathrm{m}$.

Corresponding author: svetlana_malyugina@mail.ru 
To calculate spall strength and dynamic elastic limit we used measured values of sound speed: longitudinal speed $\mathrm{c}_{1}=5964 \mathrm{~m} / \mathrm{s}$, and shear speed $\mathrm{c}_{\mathrm{s}}=3254 \mathrm{~m} / \mathrm{s}$. The calculated value of volume sound speed was $\mathrm{c}_{\mathrm{o}}=$ $4632 \mathrm{~m} / \mathrm{s}$. The density was $7.87 \mathrm{~g} / \mathrm{cm}^{3}$. To estimate the magnitude of stress in the samples under investigation we used the shock adiabat of zirconium $\mathrm{D}=4.63+14.8 \mathrm{u}$ $(\mathrm{km} / \mathrm{s})$, where D is the shock wave velocity, corrected for the obtained data on sound speed for 30KhGSA steel, $u$ is the mass velocity [6].

Shock-wave experiments were performed using a light-gas gun of 44-mm calibre [7] in the range of impact velocities from 218 to $1190 \mathrm{~m} / \mathrm{s}$. A planar shock wave was generated in the samples by strikers made of 30KhGSA steel. The striker-to-sample thickness ratio was $1 / 2$.

The velocity of striker arrival was measured with accuracy not worse than $0.4 \%$. The magnitude of nonparallelism of impacted surfaces was $1 . .2 \mathrm{mrad}$. The experiments were performed under vacuum.

In the experiment with increased and decreased sample temperature, the temperature was controlled by thermocouples with an accuracy of $\pm 5^{\circ} \mathrm{C}$.

In the experiments free surface velocity was registered by laser Doppler interferometers VISAR [8] and PDV [9]. The time resolution for VISAR interferometer was at least $2 \mathrm{~ns}$, and for PDV interferometer it was at least 4 ns. The amplitude resolution of interferometers was no more than $0.8 \%$ in the covered range of velocities.

The experiments in spall fracture incipience and development, and damage recovery (healing) were performed using two methods. The first method suggests two-stage experiments. At the first stage the set of shockwave experiments was performed with formation in the samples of different-level damages and spall fracture (from single defects up to the main crack). A correspondence between the load level and quantitative characteristics was established. At the second stage the sample with known damage level was loaded by shock waves. We also determined the changing behaviour and qualitative characteristics of the compression pulse, induced by damage compacting forces, as well as distortions of shock-wave flow caused by passing through the damaged material area.

The set-up of shock-wave experiments to study damage formation and recovery (healing) processes is given in Figure 1.

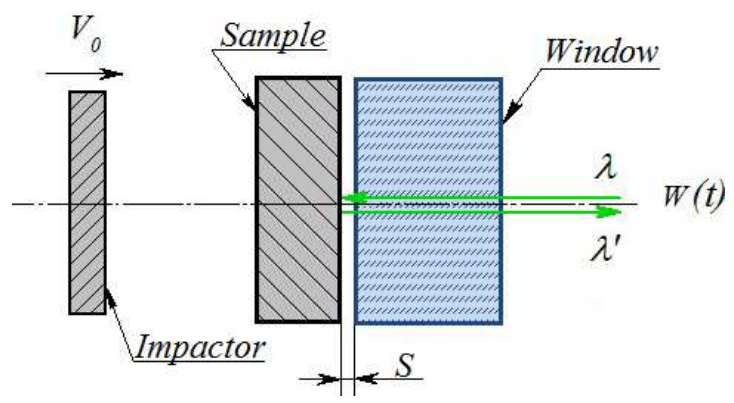

Fig. 1. The set-up of experiments to study spall fracture formation and compacting.
The impactor decelerates on the stationary sample and generates a compression wave in it. The compression waves reflected from free surfaces of the impactor and the sample turn into tension waves, with subsequent formation in the sample of tensile stresses sufficient for spall fraction incipience and development. The window made of transparent material, on which the tested sample decelerates, is positioned at a given distance $\mathrm{S}$ from the sample free surface. Thus tensile stress time and damage level, correspondingly, are limited. The rarefaction wave amplitude decay depends on the acoustical impedance ratio of the sample and the window, as well as on sample surface velocity at the impact with the receiver. Polymethylmethacrylate (PMMA), sapphire, LiF and silica glass were used as a window.

\section{Experimental results}

Figure 2 presents free surface velocity profiles for 30KhGSA steel samples of different thickness in the range of impact velocities $220 \ldots 670 \mathrm{~m} / \mathrm{s}$, which were obtained in experiments at normal temperature. Sample thickness values are given near wave profiles.

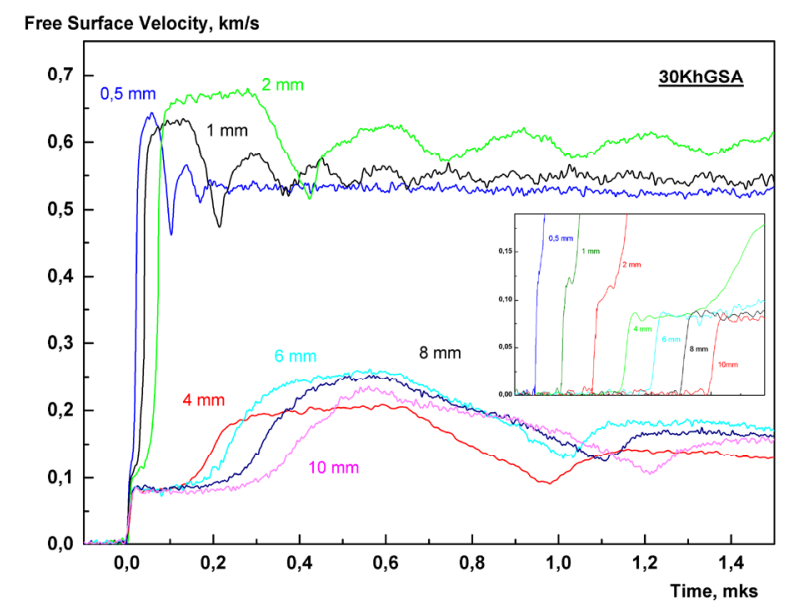

Fig. 2. Free surface velocity profiles for $30 \mathrm{KhGSA}$ steel samples.

The arrival of elastoplastic compression wave to the surface and the following rarefaction wave are registered on wave profiles. It can be seen that the velocity behind the elastic precursor front is decreased with compression wave propagation, caused by stress relaxation during plastic deformation.

Compression stress behind the elastic precursor front, that is, dynamic elastic limit $\sigma_{H E L}$, is calculated from measured free surface velocity $W_{h}$ as $\sigma_{H E L}=\rho_{0} c_{l} W_{h} / 2$.

Measurement results of elastic precursor decay in the samples of thickness from 0.5 to $10 \mathrm{~mm}$ at normal temperature are shown in Figure 3. 


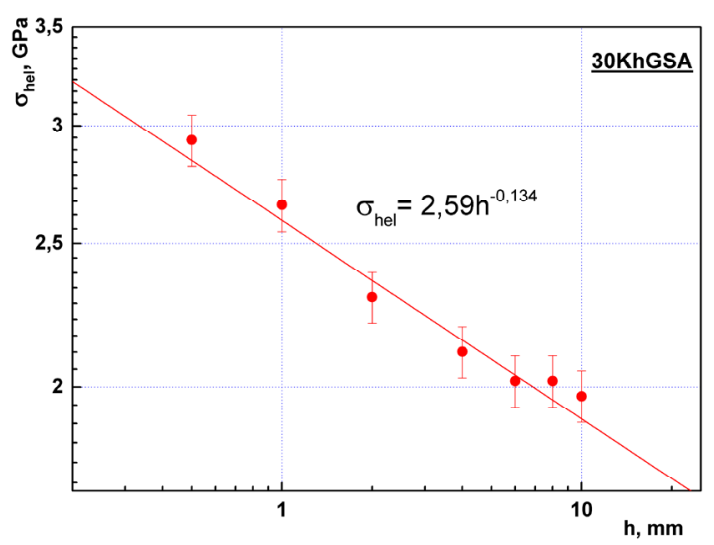

Fig. 3. Elastic precursor decay in $30 \mathrm{KhGSA}$ steel.

Experimental data are fitted with good accuracy by the empirical dependence:

$$
\sigma_{h}=2.62\left(h / h_{0}\right)^{-0.136}
$$

The investigations determined the effect of temperature on dynamic elastic limit (Figure 4).
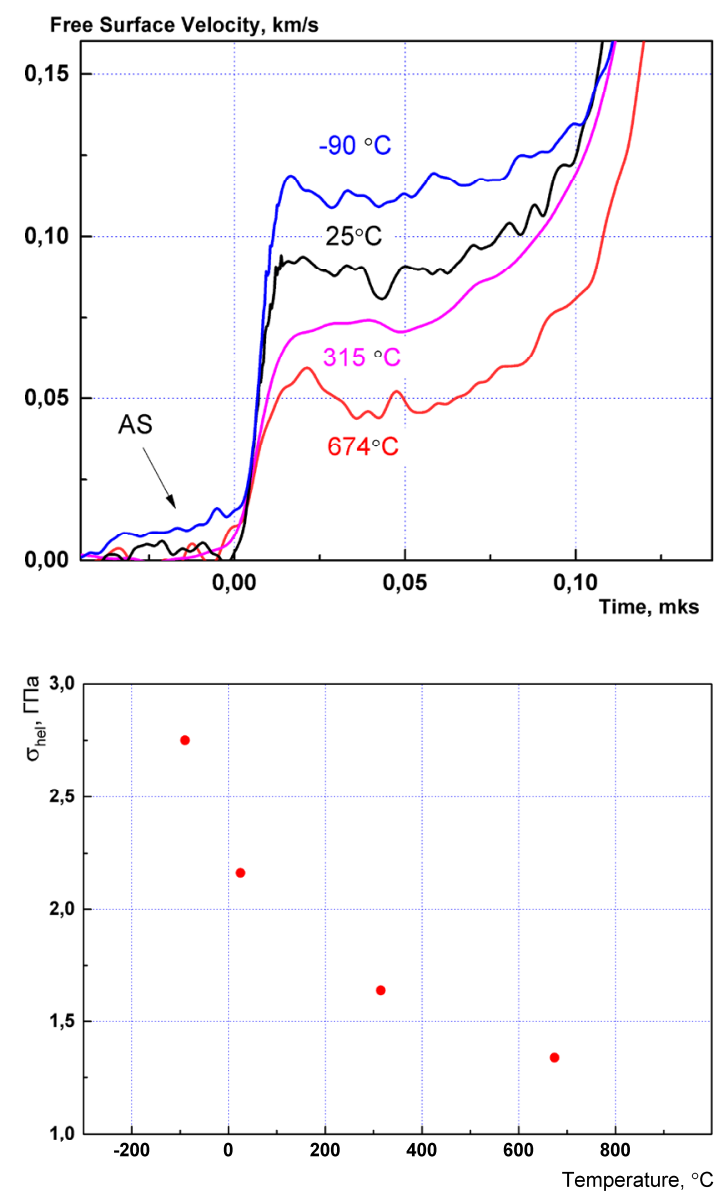

Fig. 4. Temperature effect on elastic precursor amplitude in 30KhGSA steel. The dynamic elastic limit in the range of temperatures from -90 to $670{ }^{\circ} \mathrm{C}$ is decreased monotonically.

Figure 5 presents the results of measuring stress wave profiles in 30KhGSA steel samples $4 \mathrm{~mm}$ thick at different shock wave strength. At the impact velocity up to $0.6 \mathrm{~km} / \mathrm{s}$ two-stage elastoplastic compression wave is registered. When shock compression amplitude rises, the plastic shock wave loses its stability and splits due to known polymorphous transition $\alpha \rightarrow \varepsilon$, and a phase precursor is separated in the structure of shock wave front. The estimate of phase precursor amplitude comes to $13.6 \mathrm{GPa}$. With further increase in load intensity the principle plastic wave splits with PIR-wave separation at the cost of SW reflection from phase interface.

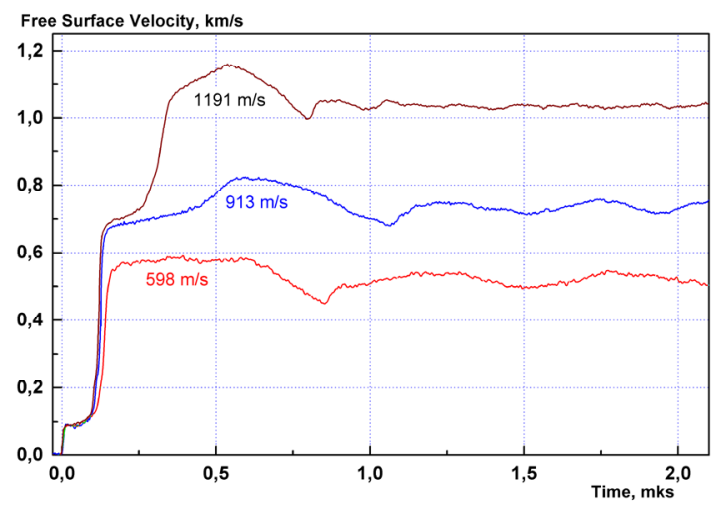

Fig. 5. Free surface velocity profiles of $30 \mathrm{KhGSA}$ steel samples. Samples are $4 \mathrm{~mm}$ thick.

The effect of temperature on phase precursor amplitude is shown in Fig. 6.

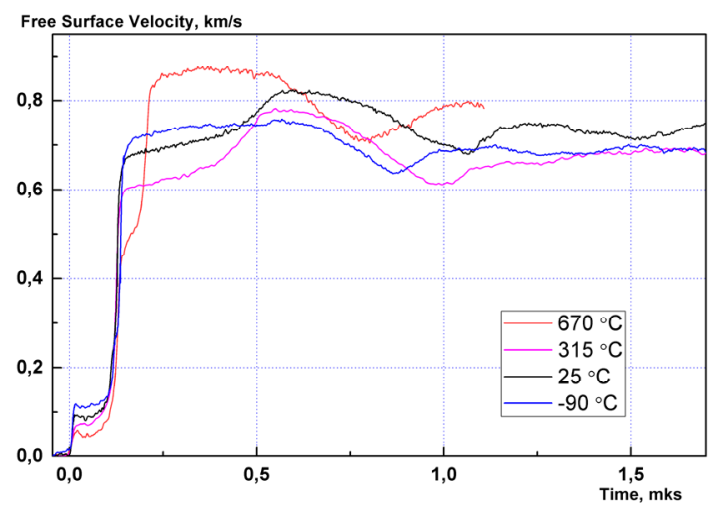

Fig. 6. Free surface velocity profiles of $30 \mathrm{KhGSA}$ steel samples with temperature variation.

As temperature increases, the start pressure of $\alpha \rightarrow \varepsilon$ phase transition drops up to $12 \mathrm{GPA}$ at $315^{\circ} \mathrm{C}$ and up to $8.7 \mathrm{GPa}$ at $670{ }^{\circ} \mathrm{C}$. When temperature is decreased up to minus $90{ }^{\circ} \mathrm{C}$ and the impact velocity comes to $786 \mathrm{~m} / \mathrm{s}$ (16 GPa) the wave splitting with phase precursor separation is not registered. Based on the obtained results on temperature effect on $\alpha \rightarrow \varepsilon$ transition in 30KhGSA steel it is assumed that load pulse pressure is insufficient to provide the onset of phase transition.

The velocity vs. spall strength of $30 \mathrm{KhGSA}$ steel was determined in the range of deformation velocities from $3.4 \times 10^{4}$ to $10^{6} \mathrm{~s}^{-1}$ (Fig. 7). The spall strength was calculated from measurement results of free surface deceleration when unloading from shocked condition corrected for velocity profile dispersion due to different 
velocities of spall pulse propagation and plastic wave in the unload area [2].

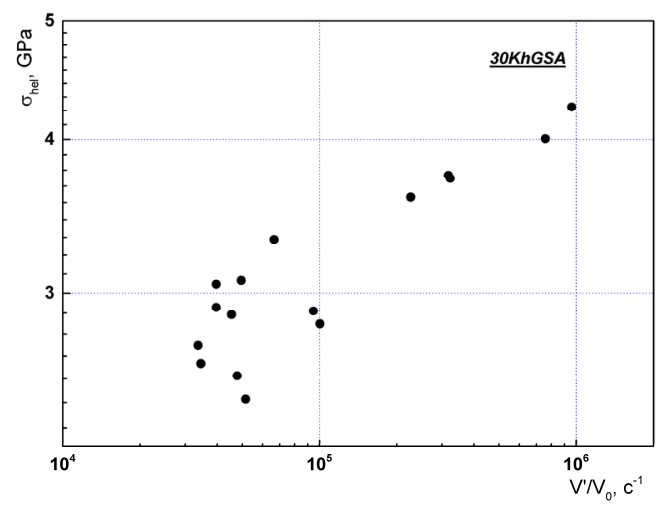

Fig. 7. Spall strength of $30 \mathrm{KhGSA}$ steel samples vs. deformation velocity.

Fig. 8 shows the temperature effect on spall strength of $30 \mathrm{KhGSA}$ steel. In computing the spall strength the variation of sound speed in the material with temperature was neglected.

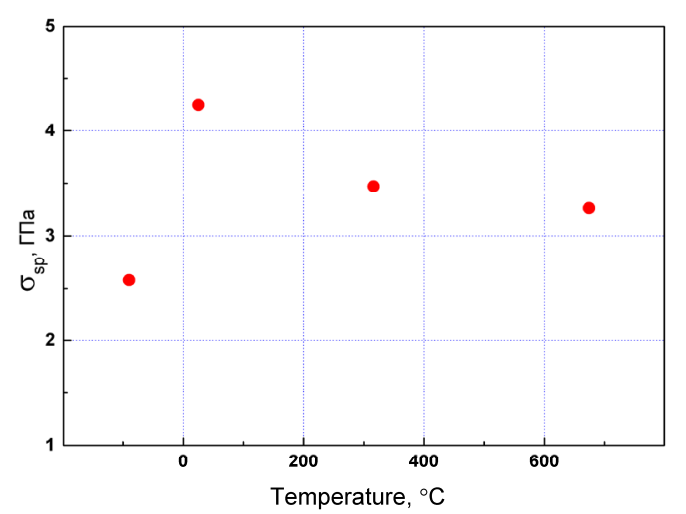

Fig. 8. Spall strength of $30 \mathrm{KhGSA}$ steel samples vs. temperature.

At lower temperature $30 \mathrm{KhGSA}$ steel demonstrates spall fracture strength decreased by $40 \%$. With temperature rising up to $670{ }^{\circ} \mathrm{C}$ the spall strength is monotonically reduced by $25 \%$.

Damage formation and recovery (healing). As was mentioned above, the investigations were performed using two methods.

Method No 1. At the first stage the set of shock-wave experiments was performed with formation in the samples of different-level damages and spall fracture. In order to form damage, from light micro-fracture up to partial spall fracture, the range of impactor velocities was varied from 228 to $420 \mathrm{~m} / \mathrm{s}$. A degree of sample damage was analysed using the methods of hydrostatic measurement of density and quantitative microscopy (Fig. 9). Using the method of hydrostatic measurement of density the integral values of porosity were determined for all samples depending on impact intensity.
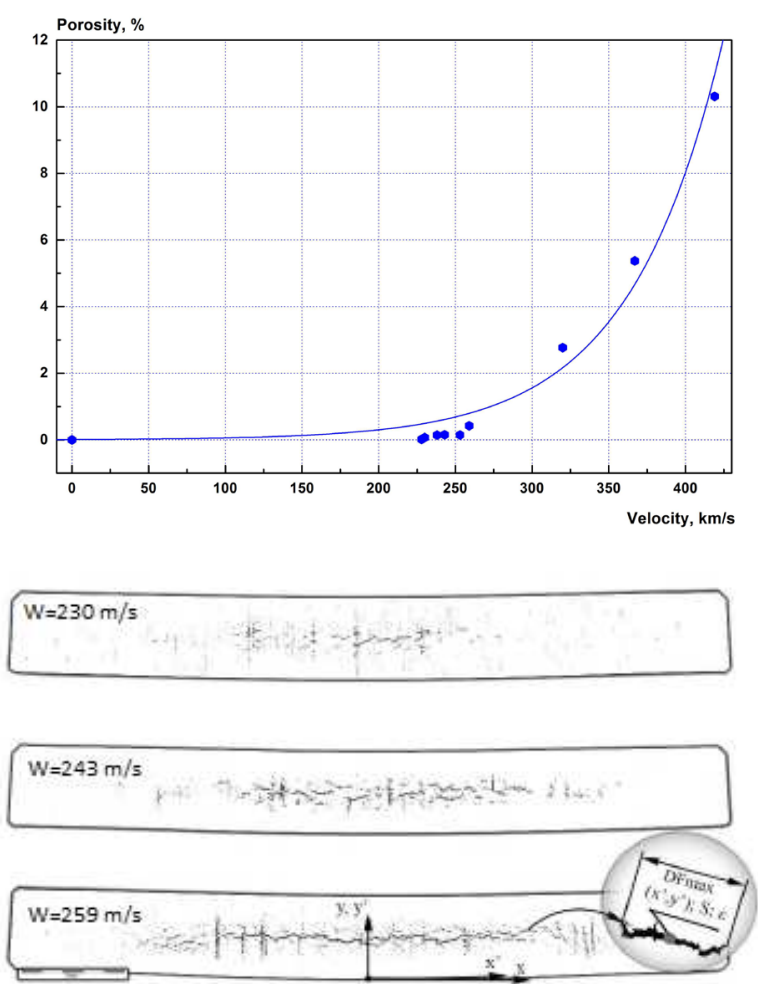

Fig. 9. Sample porosity vs. impact velocity and recovered sample microscopy.

Up to velocity of $250 \mathrm{~m} / \mathrm{s}$ the porosity varies insignificantly, suggesting the stages of light micro- and macrofracture. Beginning from impact velocity of 260 $\mathrm{m} / \mathrm{s}$ the porosity increases rather sharply, conforming microcrack merging and formation of partial spall fracture. At higher temperatures $(350 \ldots 420 \mathrm{~m} / \mathrm{s})$ a sealed main crack is formed inside the sample.

At the second stage the experiments on damage recovery (healing) were performed. Following the shockwave load the samples with different level of damages were reloaded. Two levels of damage were selected, where the porosity was equal to 0.14 and $0.27 \ldots 0.28 \%$. For loading $3.5 \mathrm{GPa}$ shock wave amplitudes were chosen, which exceed 1.3 GPa yield limit of the tested material. To prevent potential formation of additional material fracture, the velocity of gun shell with an impactor was limited by $190 \mathrm{~m} / \mathrm{s}$. The influence of sample porosity on variation of shock-wave processes is shown in Fig. 10.

The availability of damages in the sample essentially varies a profile of the wave, which arrives to its free surface. It is apparent that amplitudes of elastic precursor and plastic waves drop, and their fronts become diffused. This is due to shock wave passing through the damage area and accompanying decrease of damage level, which is time-expanded. 


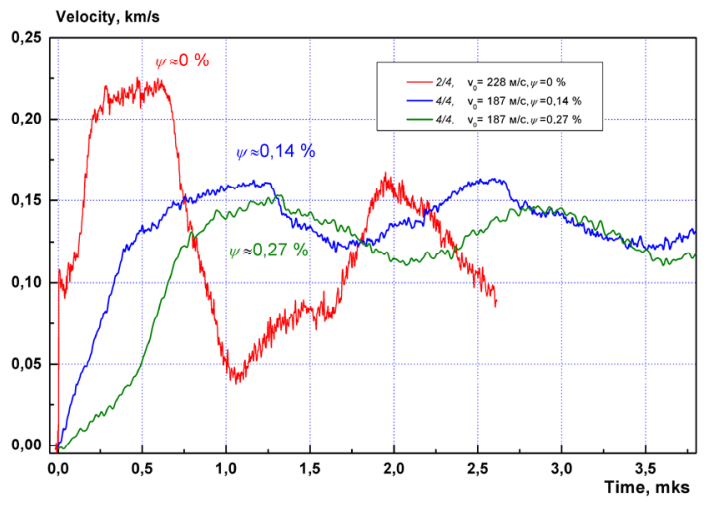

Fig. 10. Free surface velocity profiles obtained under reloading of $30 \mathrm{KhGSa}$ steel samples with different levels of damage.

The impact of the shock wave with $3.5 \mathrm{GPa}$ amplitude on the samples damaged up to $2.7 \ldots 2.8 \%$ resulted in porosity reduction by $1.5 \%$. This effect is expected and can be explained by damage recovery (healing).

Method No 2. This method allows one, in a single experiment, to initiate the process of spall fracture incipience and healing damages at the cost of the shock wave formed by sample deceleration on a transparent window (Fig. 1). The level of damage was specified by $\mathrm{S}$ gap width. Fig. 11 gives the velocity profiles obtained in experiments with the window made of PMMA.

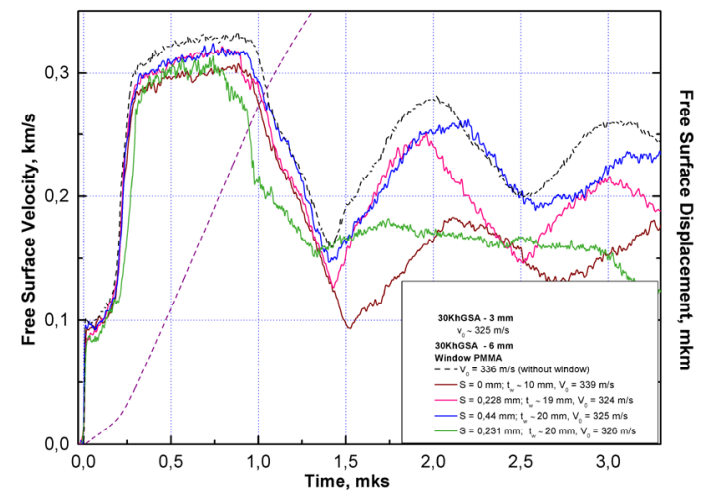

Fig. 11. Comparison of velocity profiles in experiments with PMMA windows at different $\mathrm{S}$ values.

The figure also presents the velocity profile obtained in the experiment without a window (black dashed line) and calculated free surface displacement (magenta dashed line). By the time a fracture pulse arrived to free surface, its displacement was $0.37 \mathrm{~mm}$. By this time the formation of spall fractures was mainly completed. Therefore tensile strength length was varied at the cost of $\mathrm{S}$ distance variation in the range up to $0.4 \mathrm{~mm}$.

The performed investigations into recovered samples by hydrostatic density metering method suggest the presence of internal damage in loaded samples. The metallographic analysis of the recovered samples detected multiple recovery (healing) traces. The most contrast recovery (healing) traces are presented on the sample section, which was tested at 0.44 and $0.23 \mathrm{~mm}$ distance to the window. Figure 12 shows microstructures with damage recovery (healing) traces in this sample, which represent extended smoothly curving regions comprising of incompletely closed cracks, deformed material grains and deformation bands.
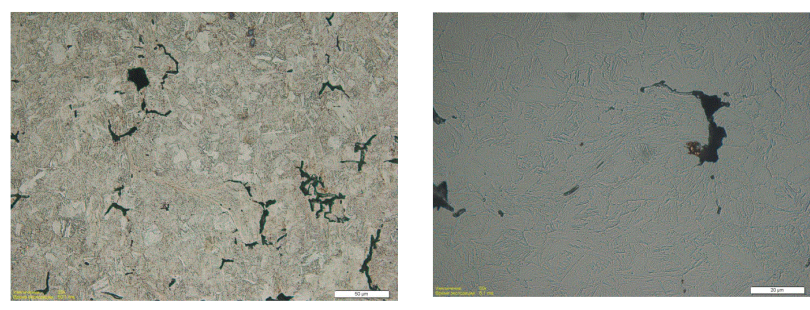

Fig. 12. Recompaction traces in experiments at $\mathrm{S}=0.44 \mathrm{~mm}$ and $\mathrm{S}=0.23 \mathrm{~mm}$.

The analysis of sample microstructure has shown that the amplitude of recovery (healing) stresses is insufficient to close spall cracks. PMMA stiffness is insufficient to effectively decelerate the spall layer and to recompact damages. Then test experiments were performed using the window made of sapphire, lithium fluoride, and KU-1 quartz optical glass. These materials have high acoustic impedance as compared to organic glass (about 9, 4 and 2.6-fold, respectively).

Fig. 13 summarizes the results of performed experiments with the windows made sapphire, lithium fluoride, and KU-1 quartz optical glass in the form of surface velocity profiles.

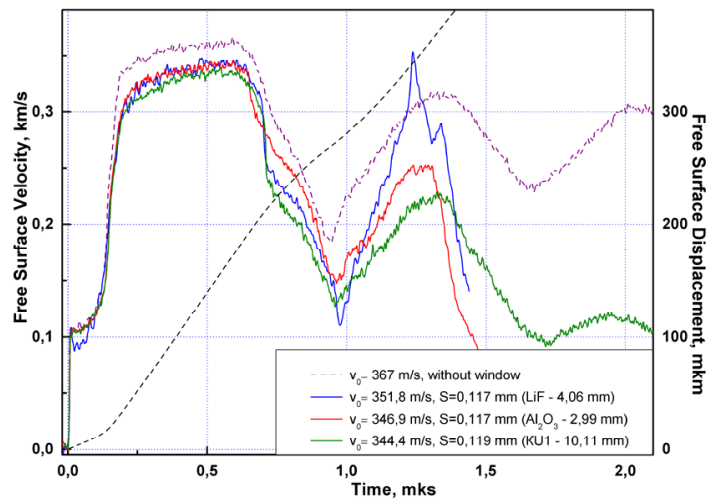

Fig. 13. Comparison of velocity profiles in experiments with different windows.

Metallographic analysis of the samples recovered in the experiments with "windows" made of sapphire, lithium fluoride, and KU-1 quartz optical glass will allow one to determine a degree of recovery (healing) for tested samples.

\section{Conclusions}

The data on strength properties of 30KhGSA alloy steel were obtained under shock-wave impact in submicrosecond range of load and pressure amplitude length in 4-26 GPA shock wave. The experiments were performed at decreased, normal, and increased temperatures.

Based on free surface velocity profiles obtained in the 
experiments, spall strength was determined, and spall strength vs. deformation rate was plotted.

The experimental data on relaxation of dynamic elastic limit in the steel samples of thickness $0.5-10 \mathrm{~mm}$ were obtained. The resulting dependencies are reasonably approximated by the empirical relation $\sigma_{h}=2.62\left(h / h_{0}\right)^{-0.136}$.

The values of shock wave amplitudes wherein phase transitions occur were determined. The estimate of phase precursor amplitude comes to $13.6 \mathrm{GPa}$. With further increase in load intensity, principle plastic wave splitting is registered with PIR-wave separation at the cost of SW reflection from phase interface.

The measurements of strength and elastoplastic properties of 30KhGSA steel were performed at elevated and reduced temperatures. The dynamic elastic limit in the range of temperatures from -90 to $670{ }^{\circ} \mathrm{C}$ is monotonically decreased. At lower temperature the spall strength is reduced by $40 \%$, and at elevated temperature up to $670{ }^{\circ} \mathrm{C}$ the spall strength is monotonically reduced by $25 \%$. Phase precursor amplitude is also drops when temperature rises.

The processes of spall fracture incipience and development, and damage recovery (healing) in 30 KhGSA steel were analysed. The data on shock wave passing through the damaged area in steel samples were obtained.

\section{References}

1. G.I. Kanel, S.V. Razorenov, A.V. Utkin, V.E. Fortov. Shock-wave phenomena in condensed media. M.: "Yanus -K", p. 408 (1996)

2. G.I. Kanel. R.J. Rus.J. PMTF. (Applied Mechanics and Technical Physics), v. 42, No 2, 1-5 (2001)
3. G.I. Kanel, S.V. Razorenov, A.V. Utkin, V.E. Fortov. Experimental profiles of shock waves in condensed matter. M.: FIZMATLIT (2008)

4. V.I. Vladimirov. Physical nature of metal fracture. Metallurgia Publisher (1984)

5. V.K. Golubev, S.A. Novikov, Yu.S.Sobolev, N.A. Yukina. On the nature of spall fracture of aluminum and its alloys D16 and Amg6 in temperature range of 196-600 ${ }^{\circ}$ C. Strength problems, No 2, pp. 53-59 (1983)

6. M.V. Zhernokletov, V.N. Zubarev, R.F. Trunin, V.E. Fortov. Experimental data on shock compressibility and adiabatic expansion of condensed matter under high energy densities. Chernogolovka Publisher (1996)

7. A.V. Pavlenko, S.I. Balabin, O.E. Kozelkov, D.N. Kazakov. Rus.J. PTE, (Instruments and experimental technique), No 4. pp. 122-124 (2013)

8. A.V. Pavlenko, S.N. Malyugina, V.V. Pereshitov, I.N. Lisitsyna. Rus.J. PTE, (Instruments and experimental technique), No 2. pp. 127-129 (2013)

9. S.S. Mokrushin, N.A. Anikin, S.N. Malyugina, A.A. Tyaktev, A.V. Pavlenko. Rus.J. PTE, (Instruments and experimental technique), No 4, pp.107-110 (2014) 